\title{
Uso de cola cirúrgica no reparo de lacerações perineais intraparto: estudo série de casos
}

Use of surgical glue to repair intrapartum perineal lacerations: a case series study Uso de pegamento quirúrgico para reparar desgarros perineales intraparto: estudio serie de casos

\author{
Adriana Caroci-Becker ${ }^{1}$ ic https://orcid.org//0000-0003-3112-8480 \\ Wesllanny Sousa Brunelli' ${ }^{1}$ (1) https://orcid.org/0000-0001-7538-1707, \\ Marlise de Oliveira Pimentel Lima' in https://oredid.org/0000-0001-5841-2188. \\ Edilaine de Paula Batista Mendes ${ }^{1}$ il hitps://orcid.org/0000-0002-8541-3490 \\ Angela Megumi Ochiai' 1 entps:///rcid.org/0000-0001-9014-876X \\ Maria Luiza Gonzalez Riesc0 ${ }^{1}$ id https://orcid.org/0000-0001-9036-5641
}

Como citar:
Caroci-Becker A, Brunelli WS, Lima MO,

Mendes EP, Ochiai AM, Riesco ML. Uso de cola cirúrgica no reparo de lacerações perineais intraparto: estudo série de casos. Acta Paul Enferm. 2021;34:eAPE02724.

DOI

http://dx.doi.org/10.37689/actaape/2021A002724

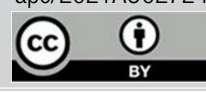

Períneo: Adesivos tecidu Cianoacrilatos; Enfermagem obstétrica

Keywords

Perineum; Tissue adhesives; Laceration Cyanoacrylates; Obstetric nursing

\section{Descriptores}

Perineo; Adhesivos tisulares; Laceración; Cianoacrilatos; Enfermería obstétrica

\section{Submetido \\ 4 de Outubro de 2019 \\ Aceito \\ 2 de Dezembro de 2020}

Autor correspondente

Adriana de Souza Caroci

E-mail: acaroci@usp.br

\section{Resumo}

Objetivo: Descrever o uso da cola cirúrgica no reparo do trauma perineal no parto normal.

Métodos: Estudo série de casos realizado em três momentos (até 2 horas, 12-24 horas e 36-48 horas após o parto), em Itapecerica da Serra, SP. Foram incluídas mulheres que tiveram parto normal com trauma perineal com indicação de sutura (laceração de primeiro ou segundo graus e episiotomia). 0 trauma perineal foi reparado exclusivamente com cola cirúrgica Glubran- ${ }^{\circledR}$. Avaliou-se: intensidade da dor perineal (Escala Visual Numérica com 11 pontos), processo de cicatrização (escala REEDA de 15 pontos), satisfação com 0 reparo (escala Likert de 5 pontos). Os dados foram analisados de forma descritiva e inferencial comparando os três momentos.

Resultados: A técnica de aplicação da cola e a quantidade necessária foram definidas em uma amostra de 19 mulheres. Destas, 78,9\% tiveram laceração de primeiro grau, 15,8\% de segundo grau e 5,3\% episiotomia. Os desfechos nos momentos 1, 2 e 3, foram respectivamente: ausência de dor (73,6\%, 94,7\% e 89,4\%); escore $\leq 1$ na escala REEDA $(94,7 \%, 78,9 \%$ e $84,2 \%) ; 100 \%$ satisfeitas com o reparo em todos os momentos. Não houve diferença pelo teste de Friedman para dor e satisfação. 0 processo de cicatrização mostrou diferença, porém sem confirmação no pós-teste hoc.

Conclusão: A aplicação da cola mostrou-se viável para avaliação em uma amostra maior de mulheres, pois os resultados sugerem boa aceitação pelas mulheres e dor de baixa intensidade ou ausente, cicatrização adequada e alta satisfação com o reparo nas primeiras 48 horas após o parto.

\section{Abstract}

Objective: To describe the use of surgical glue to repair perineal trauma during normal delivery.

Methods: This is a case series study, which was carried out in three moments (up to 2 hours, 12-24 hours and 36-48 hours after delivery) in Itapecerica da Serra, SP. Women who had a normal delivery with perineal trauma with a suture (first or second degree laceration and episiotomy) were included. Perineal trauma was repaired exclusively with Glubran- ${ }^{\circledR}$ surgical glue. Perineal pain intensity (11-point Visual Numeric Scale), healing process (15-point REEDA scale), satisfaction with repair (5-point Likert scale) were assessed. Data were analyzed in a descriptive and inferential way comparing the three moments.

Results: The technique of applying the glue and the required amount were defined in a sample of 19 women. Of these, $78.9 \%$ had first-degree lacerations, $15.8 \%$, second-degree lacerations and $5.3 \%$, episiotomy. The outcomes at moments 1, 2 and 3 were absence of pain $(73.6 \%, 94.7 \%$ and $89.4 \%)$, score $\leq 1$ on the REEDA scale $(94.7 \%, 78.9 \%$ and 84 , two\%); $100 \%$ were satisfied with the repair at all times. There was no difference by the Friedman test for pain and satisfaction. The healing process showed a difference, but without confirmation in the hoc post-test. 
Conclusion: The glue application proved to be viable for assessment in a larger sample of women, as the results suggest good acceptance by women and low or no pain, adequate healing and high satisfaction with the repair in the first 48 hours after delivery.

\section{Resumen}

Objetivo: Describir el uso de pegamento quirúrgico para reparar traumas perineales en partos vaginales.

Métodos: Estudio serie de casos realizado en tres momentos (hasta 2 horas, de 12 a 24 horas y de 36 a 48 horas después de parto), en Itapecerica da Serra, estado de São Paulo. Se incluyeron mujeres que tuvieron parto vaginal con trauma perineal e indicación de sutura (desgarro de primer 0 segundo grado y episiotomía). El trauma perineal fue reparado exclusivamente con pegamento quirúrgico Glubran- $2^{\circledR}$. Se evaluó la intensidad del dolor perineal (Escala Visual Numérica de 11 puntos), el proceso de cicatrización (Escala REEDA de 15 puntos) y la satisfacción respecto a la reparación (Escala Likert de 5 puntos). Los datos fueron analizados de forma descriptiva e inferencial, comparando los tres momentos.

Resultados: La técnica de aplicación del pegamento y la cantidad necesaria fueron definidas en una muestra de 19 mujeres. De ellas, el 78,9 \% tuvieron un desgarro de primer grado, el 15,8 \% de segundo grado y el 5,3\% episiotomía. Los resultados de los momentos 1, 2 y 3 fueron, respectivamente: ausencia de dolor (73,6 \%, 94,7 \% y 89,4 \%); puntuación $\leq 1$ en la escala REEDA (94,7 \%, 78,9 \% y 84,2 \%); 100 \% satisfechas con la reparación en todos los momentos. No se observó diferencia de dolor y satisfacción con la prueba de Friedman. El proceso de cicatrización mostró diferencia, pero sin confirmación en la prueba post hoc. Conclusión: La aplicación del pegamento demostró ser viable para un análisis con una muestra mayor de mujeres, ya que los resultados sugieren buena aceptación por parte de las mujeres, dolor de baja intensidad o ausente, cicatrización adecuada y alta satisfacción respecto a la reparación en las primeras 48 horas después del parto.

Registro Brasileiro de Ensaios Clínicos: RBR-2q5wy8

\section{Introdução}

O trauma perineal afeta mulheres em todo o mundo e mais de $85 \%$ das mulheres que tiveram um parto vaginal sofreram uma laceração perineal espontânea ou episiotomia. No Reino Unido e nos Estados Unidos, estima-se que pelo menos um terço das laceraçôes perineais requerem reparos. ${ }^{(1)}$

No Brasil, estudo de coorte observou que a ocorrência de laceraçóes perineais de primeiro e segundo graus foi de $36,4 \%$ e de laceraçóes de terceiro e quarto graus foi de $0,9 \%$. $^{(2)}$

As complicaçóes decorrentes do trauma perineal no processo de cicatrização podem gerar morbidades físicas e psicológicas em curto e longo prazo como: dor, edema, hiperemia, equimose, infecção, deiscência, dispareunia, incontinência urinária, incontinência anal e diminuição da força muscular do assoalho pélvico (FMAP). ${ }^{(3-5)}$

A recuperação do períneo e a redução das morbidades dependem da prevenção e da avaliação correta do trauma, da técnica utilizada para o reparo e da escolha do material. ${ }^{(6-9)}$ A sutura com fio é a técnica mais tradicional para o reparo perineal no parto normal, sendo que o fio mais utilizado é o categute, contudo, estudos consideram que a técnica de sutura contínua utilizando fio sintético Vicryl de rápida absorção é a mais recomendada para o reparo do períneo. ${ }^{(6,9)}$

A cola cirúrgica tem sido testada para o reparo de trauma tecidual em diversas áreas da saúde, por ser menos invasiva e por apresentar um elevado grau de resistência, facilitando o procedimento cirúrgico. ${ }^{(10-12)}$

Estudo comparativo entre o uso do fio de sutura $\mathrm{Vicryl}^{\circ}$ de rápida absorção e a cola cirúrgica octy1-2-cyanoacrylate, usada em traumas superficiais, constatou que o uso da cola para o reparo perineal apresentou resultados estéticos e funcionais similares ao da sutura com fio e ainda apresentou vantagens, como redução no tempo do procedimento de reparo, diminuição da dor, isenção da necessidade da anestesia local e maior satisfação da mulher. ${ }^{(13)}$

Em estudo piloto paralelo, controlado e aleatorizado, que comparou o uso da cola cirúrgica com o fio de sutura em laceraçóes de primeiro grau foram verificados menor intensidade de dor, melhor escore de cicatrização e maior satisfação em mulheres submetidas ao reparo com cola cirúrgica do que com fio de sutura. ${ }^{(14)}$

Outro ensaio clínico aleatorizado com 100 primigestas comparou o reparo da pele em episiotomias, utilizando em um grupo a cola Dermabond ${ }^{\circ} \mathrm{e}$ no outro o fio Vicryl Rapide; com o uso da cola observaram-se menor tempo de reparo e menor intensidade da dor durante e após o procedimento, sendo semelhantes os resultados relativos à cicatrização. ${ }^{(15)}$

Acredita-se que o reparo perineal com cola cirúrgica pode contribuir para a maior satisfação da mulher com o parto normal, pois pode diminuir a intensidade da dor perineal e melhorar o processo de cicatrização perineal. 
A realização deste trabalho justifica-se pela necessidade de estudar mais amplamente o uso da cola cirúrgica no trauma perineal, pois embora a literatura mostre resultados positivos em obstetrícia e em outras áreas da saúde, não há estudos publicados sobre o reparo com cola em todos os planos teciduais, ou seja, em laceraçóes de segundo grau e/ou em episiotomias, além de existir uma variedade de tipos de cola e falta de informaçóes sobre a melhor técnica de aplicação no reparo perineal.

Nesse contexto, visando conhecer novas técnicas e materiais para aprimorar a assistência ao parto e a qualidade no cuidado do trauma perineal em laceraçóes de primeiro e segundo graus ou episiotomias, este estudo teve como objetivo descrever o uso da cola no reparo do trauma perineal decorrente de parto normal.

\section{Métodos}

\section{Tipo de estudo, local e amostra}

Trata-se de um estudo série de casos sobre o uso da cola cirúrgica Glubran- $2^{\circ}$ no reparo perineal de laceraçóes perineais de primeiro e segundo graus ou episiotomia, em relação à dor perineal, processo cicatricial e satisfação das mulheres, com seguimento até 48 horas após o parto normal. $\mathrm{O}$ estudo foi realizado no Centro do Parto Normal (CPN) do Pronto Socorro e Maternidade Municipal Zoraide Eva das Dores (PSMMZED), referência para gestantes de risco habitual, no município de Itapecerica da Serra, São Paulo.

A amostra foi por conveniência com 19 mulheres que aceitaram ser submetidas ao reparo perineal com cola cirúrgica e atenderam aos seguintes critérios de inclusão: estar em trabalho de parto com até 6 centímetros de dilatação; não ter parto vaginal anterior; não fazer uso de substâncias esteroides; não apresentar leucorréia ou qualquer sinal de infecção no local de reparo; não apresentar dificuldade no entendimento do idioma português ou na comunicação.

Os critérios de exclusão foram a indicação de cesárea no decorrer do trabalho de parto e parto normal com períneo íntegro ou com trauma perineal sem indicativo de sutura. Ressalta-se que a necessidade ou náo do reparo perineal/sutura era avaliada por um profissional do serviço que não fazia parte da equipe de pesquisa e a avaliaçáo do reparo perineal era realizada por um enfermeiro da equipe de pesquisa.

\section{Descrição sobre a cola cirúrgica utilizada: Intervenção}

Para o reparo perineal foi utilizada exclusivamente a cola cirúrgica sintética Glubran- $2^{\circ}$, de base cianoacrílica, modificada pela adição de um monômero, sintetizado pelo próprio fabricante GEM S.r.l, na Itália. Esta cola é classificada como um produto médico cirúrgico de classe III, a qual pode ser utilizada na pele e em tecidos superficiais e profundos, conforme os requisitos da Diretiva Europeia 93/42/CEE. Destaca-se que essa cola possui registro (80159010003) na Agência Nacional de Vigilância Sanitária (ANVISA). O fabricante descreve ainda que a cola é versátil, pois pode ser utilizada em todos os tipos de cirurgias tradicionais, laparoscópicas e tratamentos endoscópios, além de ter altas propriedades hemostáticas e adesivas. Uma vez solidificada, a cola produz uma barreira antisséptica eficaz contra os agentes infecciosos ou patogênicos mais frequentes nas intervençôes cirúrgicas. É um líquido claro, transparente e pronto para ser utilizado.

Quando em contato com tecido vivo ou ambiente úmido polimeriza rapidamente, criando uma fina película elástica com elevada resistência à tensão, o que garante uma sólida adesão dos tecidos e não é danificada pelo sangue ou fluidos orgânicos. O tempo de polimerização varia em função do tipo de tecido que a cola entra em contato, da natureza dos fluidos presentes e da quantidade de produto aplicado. Se utilizada corretamente, a cola inicia sua solidificação em, aproximadamente, 1 a 2 segundos, completando a sua reação após cerca de 60 a 90 segundos, alcançando sua máxima resistência mecânica. Nos processos cirúrgicos normais, a película de cola é eliminada mediante processo de degradação hidrolítica. A duração deste processo varia conforme o tipo de tecido e a quantidade de cola aplicada.

Devido o tipo de intervenção e dos desfechos, não houve possibilidade de ocorrer o cegamento, por isso tanto as participantes como as pesquisadoras sabiam durante todo o tempo que o tipo de reparo perineal seria com a cola cirúrgica. As mulheres também sa- 
biam que seriam acompanhadas pelas pesquisadoras durante toda a internação. Também vale mencionar que durante a avaliação do processo de cicatrização, é possível verificar o material utilizado.

\section{Variáveis, exposição e desfechos}

As variáveis analisadas foram: intensidade da dor perineal, processo cicatricial e satisfação da mulher com o reparo perineal realizado.

Foi considerado como exposição o reparo perineal com cola cirúrgica e como desfechos considerou-se a intensidade da dor, a cicatrização perineal e a satisfação da mulher com o reparo perineal.

A intensidade da dor perineal foi avaliada mediante o uso da EVN, que consiste em uma linha horizontal com 11 pontos e valores em centímetros de 0 (zero) a 10 (dez), sendo 0 classificado como sem dor e 10 a pior dor possível. ${ }^{(16)}$ A participante do estudo recebeu a escala e apontou o número correspondente à intensidade da dor. Para fins de análise estatística, a dor foi categorizada, posteriormente, como: sem dor (0), dor leve (1-4), moderada (5-7) e dor intensa (8-10). ${ }^{(17)}$

Para avaliar o processo de cicatrização do trauma perineal foi utilizada a escala REEDA que corresponde a cinco itens em inglês: redness, edema, ecchymosis, discharge, aproximation e em português significa: hiperemia, edema, equimose, secreção e coaptação. Para cada item avaliado foi atribuído uma pontuação de 0 (zero) a 3 (três), sendo que a pontuação máxima de 15 corresponde ao pior resultado de cicatrização perineal. ${ }^{(18)}$ No entanto, a literatura não apresenta um valor de pontuação definido para se afirmar que o processo de reparação perineal já ocorreu; o consenso é que valores ao redor de 1 são indicativos de que o processo cicatricial está ocorrendo ou já ocorreu de forma adequada. Para mensurar os itens da escala REEDA, foi utilizada a régua Peri-Rule. Essa ferramenta é de plástico maleável, graduada em centímetros, e é recomendada para mensurar a profundidade e a extensão do trauma perineal. ${ }^{(19)}$ Essa régua foi envolvida em uma camada de filme PVC e reutilizada após higienização com água e sabão, seguida por antissepsia com álcool a 70\%. Em cada avaliação, a pesquisadora verificava a necessidade ou não de novo reparo perineal e caso o item de coaptação fos- se maior ou igual a dois, foi realizado novo reparo com cola cirúrgica ou com fio de sutura caso houvesse dificuldade com o reparo com cola cirúrgica.

O nível de satisfação da mulher com o tipo de reparo perineal foi avaliado através de escala Likert de cinco pontos, correspondendo a: muito insatisfeita, insatisfeita, indiferente, satisfeita e muito satisfeita. Para o relato da satisfação com o reparo perineal foi solicitado que a mulher visualizasse a região do reparo perineal, através de um espelho, e, após, respondesse sobre sua satisfação.

\section{Treinamento, descrição da técnica utilizada para aplicação da cola cirúrgica Glubran-2@ e coleta de dados}

Antes do início da coleta de dados, realizou-se a aplicação da cola cirúrgica em tecidos de língua bovina e outras peças de carne bovina, como a ponta de paleta e o acém, a fim de desenvolver e aprimorar a técnica. Após a definição da melhor técnica de aplicação da cola cirúrgica, foi realizado o treinamento de toda a equipe de pesquisa, ainda em peças bovinas, para padronizar o modo de aplicação. A partir desse momento, iniciou-se a utilização da cola cirúrgica nas mulheres incluídas na pesquisa, sendo realizados os ajustes no procedimento, conforme se mostrasse necessário, até o estabelecimento da técnica definitiva de aplicação. Vale salientar que uma enfermeira e uma bióloga, que eram representantes da distribuidora da cola, acompanharam as pesquisadoras durante o treinamento, objetivando auxiliar na aplicação da técnica.

As laceraçóes perineais irregulares ou a presença de sangramento ativo tornam o reparo mais difícil do que em laceraçóes regulares ou sem sangramento, mesmo no reparo com fio cirúrgico. Este fato também foi observado com o uso da cola cirúrgica. Contudo, as pesquisadoras acreditam que o aprimoramento da técnica de aplicação da cola relativa à quantidade, tempo de polimerização e utilização de aplicador ou de seringa e agulha demandou um tempo maior do estudo, que o esperado inicialmente, até que a técnica fosse desenvolvida com excelência. Vale esclarecer que a descrição da técnica utilizada para aplicação da cola cirúrgica foi aprimorada durante a coleta de dados desse estudo, uma vez que já havia sido testada em tecidos bovinos. 
A técnica foi desenvolvida e as pesquisadoras propuseram um protocolo para aplicação da cola cirúrgica no reparo perineal, conforme descrito a seguir:

1. Colocar a mulher na posição ginecológica, com a região genital desnuda;

2. Calçar luvas estéreis;

3. Avaliar as condiçóes do períneo e classificar as laceraçóes perineais ou episiotomia;

4. Introduzir um chumaço de gazes no introito vaginal para impedir a saída de sangue e manter a região do trauma perineal seca, se necessário;

5. Fazer antissepsia com soro fisiológico a $0,9 \%$ no local onde será feito o reparo perineal;

6. Secar com gazes a região a ser reparada;

7. Deixar defluir o produto presente do pescoço da ampola ao fundo desta última;

8. Verificar o estado de fluidez e transparência da cola. Se o produto apresentar um aspecto pouco fluido e/ou turvo, náo poderá ser utilizado;

9. Remover o tampo da ampola, girando-o;

10. Inverter a ampola e, com uma pressão ligeira, deixar defluir a cola até impregnar a extremidade da ampola;

11. Exercer uma ligeira pressão no corpo da ampola, aplicar a cola cirúrgica diretamente no tecido subcutâneo, músculos, pele e mucosa vaginal, gota a gota, sendo aproximadamente uma gota por $\mathrm{cm}^{2}$;

12. Aproximar os tecidos, com os dedos das mãos, obedecendo a anatomia perineal, sustentando as bordas com dois dedos por 90 segundos, período necessário para que a cola complete a sua reação;

13. Verificar se o reparo perineal está adequado, ou seja, de acordo com a anatomia perineal e caso não esteja adequado, desfazer a colagem com uma lâmina de bisturi ou pinça e tentar nova aplicação, caso não seja possível, deve-se suturar a laceração por técnica contínua e com o fio Vicryl de rápida absorção;

14. Deixar a mulher em uma posição confortável e coberta.

15. Orientar quanto a realização de higiene com água e sabão no local reparado com cola cirúrgica, no mínimo três vezes ao dia, e orientar que caso percebesse a presença da cola, não deveria removê-la.
A coleta dos dados se deu em três momentos, nos meses de fevereiro a março de 2017:

No Momento 1 foi realizado durante o trabalho de parto, na fase de elegibilidade, quando as mulheres eram convidadas a participar da pesquisa, após explanação dos objetivos do estudo. Realizouse a entrevista para o levantamento dos dados sociodemográficos e obstétricos. Após o nascimento, as mulheres que atenderam aos critérios de inclusão foram inseridas no estudo e foi realizada a aplicação da cola cirúrgica para o reparo perineal. Imediatamente após o reparo perineal, em um período de no máximo 2 horas após o parto foram coletadas as demais variáveis do estudo (dor, cicatrização perineal e satisfação da mulher).

O Momento 2 ocorreu entre 12 e 24 horas após o parto e o Momento 3 entre 36 e 48 horas após o parto.

A coleta de dados foi realizada por seis enfermeiras obstetras que faziam parte da equipe de pesquisa e qualquer uma dessas profissionais poderia realizar o reparo perineal. A avaliação do reparo perineal era feita por uma dessas enfermeiras pesquisadoras em conjunto com a enfermeira de plantáo, sem a exigência de que a avaliação da mulher fosse feita por uma única profissional durante os três momentos. Dessa forma, a mulher poderia ser avaliada por profissionais diferentes ao longo dos três momentos de coleta de dados.

\section{Tratamento estatístico}

A análise dos dados foi realizada por estatística descritiva e inferencial, por teste não paramétrico de Friedman, com comparação entre os três momentos. Foi adotado o valor de $\mathrm{p}=0,05$ para significância.

\section{Aspectos éticos}

$\mathrm{O}$ projeto de pesquisa foi aprovado pelo Conselho Municipal de Saúde de Itapecerica da Serra e pelo Comitê de Ética em Pesquisa da Escola de Artes, Ciências e Humanidades da Universidade de São Paulo (CAAE 44832615.1.0000.5390). O estudo seguiu todas as determinaçóes éticas, com a participação voluntária das mulheres na pesquisa, demonstrada pela assinatura no Termo de Consentimento Livre e Esclarecido. Vale mencionar que as pesquisadoras náo têm nenhum tipo de vínculo com os fabricantes ou distribuidores dos materiais utilizados neste estudo. 


\section{Resultados}

Foram abordadas no estudo inicialmente 28 mulheres que atendiam aos critérios de inclusão durante o trabalho de parto, contudo houve a perda de uma mulher por desistência em participar da pesquisa e oito foram excluídas da amostra final na sequência. Os motivos que levaram à exclusão foram: duas mulheres tiveram indicação de cesariana; quatro apresentaram trauma perineal sem necessidade de reparo e em dois casos houve sangramento perineal excessivo (uma após laceração de segundo grau e outra após episiotomia), ocorrendo dificuldade no reparo com cola, uma vez que a polimerização se dava antes da aderência entre os tecidos, sendo necessário o reparo com fio de sutura. Portanto, foi observado que a presença de sangramento abundante, com necessidade de hemostasia local por pressão, em tecido a ser reparado impedia o uso da cola cirúrgica, verificando-se uma taxa de $9,5 \%$ $(\mathrm{n}=2)$ de insucesso com o procedimento. Não foram observados outros eventos que impedissem a utilização da cola cirúrgica para o reparo perineal. A amostra final foi composta por 19 puérperas avaliadas desde o reparo perineal até 48 horas após o parto.

\section{Características sociodemográficas e obstétricas da amostra de mulheres}

A média da idade das mulheres foi de 20 anos (d.p.=6,8); 52,6\% ( $\mathrm{n}=10)$ se autodenominaram pardas ou pretas e $47,4 \%(n=9)$ brancas; a maioria concluiu o ensino médio $(63,2 \% ; n=12) ; 26,3 \%(n=5)$ exerciam atividade remunerada; todas relataram ter parceiro fixo; a média da idade gestacional na internação foi de 39,6 (d.p.=1,1) semanas; Quanto às condiçốes do períneo, 78,9\% $(\mathrm{n}=15)$ tiveram laceraçôes de primeiro grau, $15,8 \%(n=4)$ de segundo grau e uma episiotomia, $5,3 \%$, com maior ocorrência na regiáo da fúrcula $(73,6 \% ; n=14)$ e vestibular $(42,1 \% ; n=8)$ (dados não apresentados em tabela).

\section{Dor perineal, cicatrização e satisfação das mulheres}

A ausência de dor perineal, avaliada pela EVN, foi referida pela maioria das participantes, nos três momentos. Apenas duas mulheres referiram dor moderada ou intensa logo após o reparo perineal. $\mathrm{O}$ teste de Friedman não mostrou diferença significativa entre os três momentos com relação à intensidade da dor $(\mathrm{p}=0,142)$ (Tabela 1$)$.

Em relação ao processo de cicatrização, avaliado pela escala REEDA, apenas uma mulher apresentou alteração (escore 2) logo após o reparo perineal. No entanto, metade delas teve escore de 1 a 3 entre 12 e 24 horas pós-parto. Entre 36 e 48 horas pós-parto, houve melhora no escore. $\mathrm{O}$ teste de Friedman mostrou diferença entre os três momentos $(\mathrm{p}=0,014)$, porém sem confirmação no pós-teste hoc, o que permite inferir que não houve diferença significativa (Tabela 1). Ao realizar o pós-teste hoc (significância $0,05 / 3$ momentos $=0,016$ ) considerou-se o valor 0,014 muito próximo ao corte de significância, não sendo atribuído diferença entre os grupos. A satisfação das participantes com o reparo perineal foi progressiva nas 48 horas pós-parto, e nos momentos 2 e 3 todas as mulheres referiram estar satisfeitas ou muito satisfeitas. Não houve diferença significativa entre os três momentos do estudo ( $\mathrm{p}=0,526)$ (Tabela 1$)$.

Tabela 1. Intensidade da dor, processo de cicatrização e a satisfação das mulheres com reparo perineal

\begin{tabular}{|c|c|c|c|c|}
\hline Intensidade da dor & $\begin{array}{c}\text { Momento } 1 \\
\mathrm{n}(\%)\end{array}$ & $\begin{array}{c}\text { Momento } 2 \\
n(\%)\end{array}$ & $\begin{array}{c}\text { Momento } 3 \\
\mathrm{n}(\%)\end{array}$ & $p$-value \\
\hline Sem dor & $14(73,6)$ & $18(94,7)$ & $17(89,5)$ & $0,142^{*}$ \\
\hline Dor leve (1-4) & $3(15,8)$ & $1(5,3)$ & $2(10,5)$ & \\
\hline Dor moderada (5-7) & $1(5,3)$ & $-(-)$ & $-(-)$ & \\
\hline Dor intensa (8-10) & $1(5,3)$ & $-(-)$ & $-(-)$ & \\
\hline Total & 19(100) & $19(100)$ & 19(100) & \\
\hline Escore da Escala REEDA & $\begin{array}{c}\text { Momento } 1 \\
\mathrm{n}(\%)\end{array}$ & $\begin{array}{c}\text { Momento } 2 \\
\mathrm{n}(\%)\end{array}$ & $\begin{array}{c}\text { Momento } 3 \\
\mathrm{n}(\%)\end{array}$ & \\
\hline 0 & $18(94,7)$ & $9(47,3)$ & $12(63,2)$ & $0,014^{*+}$ \\
\hline 1 & $-(-)$ & $6(31,6)$ & $4(21,0)$ & \\
\hline 2 & $1(-)$ & $3(15,8)$ & $3(15,8)$ & \\
\hline 3 & $-(-)$ & $1(5,3)$ & $-(-)$ & \\
\hline Total & $19(100)$ & $19(100)$ & $19(100)$ & \\
\hline Satisfação da mulher & $\begin{array}{c}\text { Momento } 1 \\
\mathrm{n}(\%)\end{array}$ & $\begin{array}{c}\text { Momento } 2 \\
\mathrm{n}(\%)\end{array}$ & $\begin{array}{c}\text { Momento } 3 \\
\mathrm{n}(\%)\end{array}$ & \\
\hline Muito insatisfeita & $1(5,3)$ & $-(-)$ & $-(-)$ & $0,526^{*}$ \\
\hline Insatisfeita & $-(-)$ & $-(-)$ & $-(-)$ & \\
\hline Indiferente & $1(5,3)$ & $-(-)$ & $-(-)$ & \\
\hline Satisfeita & $7(36,8)$ & $10(52,6)$ & $7(36,8)$ & \\
\hline Muito satisfeita & $10(52,6)$ & $9(47,4)$ & $12(63,2)$ & \\
\hline Total & 19(100) & 19(100) & 19(100) & \\
\hline
\end{tabular}

*Teste de Friedman; +Pós teste Hoc

$\mathrm{Na}$ avaliação dos cinco itens da escala REEDA (Tabela 2), verificou-se que o edema, a equimose e a coaptação foram os parâmetros com alguma alteração. $\mathrm{O}$ edema perineal ou vulvar entre 1 e $2 \mathrm{~cm}$ 
da incisão (escore 2) foi observado em uma mulher no primeiro momento, enquanto o edema perineal com menos de $1 \mathrm{~cm}$ a partir da incisão (escore 1 ) foi verificado em quatro mulheres no segundo momento. A equimose até $0,25 \mathrm{~cm}$ da incisão bilateralmente ou $0,5 \mathrm{~cm}$ unilateralmente (escore 1 ), foi verificada em uma mulher no segundo momento. A coaptação irregular das bordas, com pele separada a $3 \mathrm{~mm}$ ou menos (escore 1), foi visualizada em duas mulheres no segundo momento e em quatro mulheres no terceiro momento. Por sua vez, a separação da pele e subcutâneo (escore 2) ocorreu em quatro mulheres no segundo momento e em três mulheres no terceiro momento.

Tabela 2. Itens da escala REEDA com alteração

\begin{tabular}{|c|c|c|c|c|c|c|c|}
\hline \multirow[b]{2}{*}{ Pontuação } & \multicolumn{6}{|c|}{ Edema } & \multirow{2}{*}{$\begin{array}{l}\text { Total } \\
\mathrm{n}(\%)\end{array}$} \\
\hline & 0 & $\%$ & 1 & $\%$ & 2 & $\%$ & \\
\hline Momento 1 & 18 & 94,7 & - & - & 1 & 5,3 & $19(100,0)$ \\
\hline Momento 2 & 15 & 78,9 & 4 & 21,0 & - & - & $19(100,0)$ \\
\hline \multirow[t]{2}{*}{ Momento 3} & 19 & 100,0 & - & - & - & - & $19(100,0)$ \\
\hline & \multicolumn{6}{|c|}{ Equimose } & Total \\
\hline Pontuação & 0 & $\%$ & 1 & $\%$ & 2 & $\%$ & $\mathrm{n}(\%)$ \\
\hline Momento 1 & 19 & 100,0 & & & & & \\
\hline Momento 2 & 18 & 94,7 & 1 & 5,3 & - & - & $19(100,0)$ \\
\hline \multirow[t]{2}{*}{ Momento 3} & 19 & 100,0 & - & - & - & - & $19(100,0)$ \\
\hline & \multicolumn{6}{|c|}{ Coaptação } & Total \\
\hline Pontuação & 0 & $\%$ & 1 & $\%$ & 2 & $\%$ & $n(\%)$ \\
\hline Momento 1 & 19 & 100,00 & - & - & - & - & $19(100,0)$ \\
\hline Momento 2 & 13 & 68,4 & 2 & 10,5 & 04 & 21,1 & $19(100,0)$ \\
\hline Momento 3 & 12 & 63,2 & 04 & 21,1 & 03 & 15,7 & $19(100,0)$ \\
\hline
\end{tabular}

$\mathrm{Na}$ aplicação da cola cirúrgica foi observado que uma quantidade excessiva de cola impedia a aproximação adequada dos tecidos, por formar uma película não aderente. Dessa forma, a técnica gota a gota demonstrou-se a ideal.

\section{Discussão}

Com base nos resultados apresentados, foi verificado que a técnica de aplicaçáo da cola cirúrgica descrita se mostrou factível para ser utilizada em futuros estudos. Sugere-se o treinamento prévio dos profissionais para a realização da técnica de aplicação da cola cirúrgica para o reparo perineal, pois embora seja um método simples, requer treino e habilidade.

Os resultados desse estudo demonstram também que a realização de pesquisas utilizando a cola cirúrgica é viável para o reparo perineal em laceraçóes de primeiro e segundo graus e episiotomia, pois houve baixa intensidade de dor, adequado processo cicatricial do trauma perineal e boa satisfaçáo das mulheres com o procedimento, com resultados semelhantes aos encontrados na literatura com o uso do fio de sutura. ${ }^{(13,20-22)}$

A técnica de sutura contínua com o fio de sutura Vicryl de rápida absorção têm sido apresentados como padrão ouro para o reparo perineal por resultarem em diminuição da dor perineal, melhor cicatrização do trauma perineal e maior satisfação da mulher com o reparo perineal. ${ }^{(20)}$

$\mathrm{Na}$ literatura, verifica-se que estudos comparativos entre o uso da cola cirúrgica e fio de sutura para o reparo perineal demonstraram que foram menores os escores de intensidade de dor com a utilização da cola cirúrgica. ${ }^{(21)}$ No estudo atual, não foi comparado o uso da cola cirúrgica com o fio de sutura, no entanto, verificaram-se poucos relatos de dor perineal logo após o reparo perineal e nenhum relato entre 12 e 48 horas, indicando que, assim como em outras pesquisas, parece haver diminuição da intensidade da dor perineal nos dois primeiros dias pós-parto com o uso da cola. ${ }^{(21,22)}$

Em ensaio clínico aleatorizado sobre o uso da cola cirúrgica ou sutura com fio para o reparo de laceraçóes de primeiro grau foi observado um escore significantemente menor na EVN de dor com o uso da cola $(1,7$ versus 4,$1 ; \mathrm{p}>0,001)$ imediatamente após o procedimento. A satisfação das mulheres com relação aos dois procedimentos foi elevada nos dois procedimentos de reparo perineal (média de 9,42 em uma escala de 0 a 10 , sendo 10 muito satisfeita). Os autores concluíram que, além da diminuição da intensidade da dor, o uso da cola apresentou resultados estéticos e funcionais similares ao da sutura com fio e apresentou vantagens como redução no tempo de reparo perineal, isenção de necessidade de anestesia local e maior satisfação da mulher. ${ }^{(13)}$

Quando avaliado o processo de cicatrização pela escala REEDA, foram identificados apenas os itens edema, equimose e coaptação, contudo sem diferença significativa entre os três momentos observados. Em diferentes estudos, ao se comparar os métodos de sutura convencional e o uso da cola cirúrgica, verificou-se que não houve diferença no processo de 
cicatrização entre os dois métodos, no entanto, os autores concluíram que a eficácia, a qualidade e a segurança no processo de cicatrização ao utilizar a cola cirúrgica foram maiores. ${ }^{(15,22)}$

Optou-se por avaliar a satisfaçáo das mulheres em relação ao procedimento, pois os estudos realizados na área da saúde têm-se preocupado cada vez mais com a satisfação do indivíduo, por considerarem que os dados coletados são significativos para identificar lacunas e desenvolver melhorias na assistência, na escolha dos materiais utilizados e para atender melhor às expectativas das usuárias. ${ }^{(23)}$

A satisfação das mulheres em relação ao reparo com a cola cirúrgica foi observada em todos os momentos da pesquisa, mostrando boa aceitação das mulheres quanto ao aspecto do seu períneo e quanto ao uso da cola cirúrgica, confirmando os achados encontrados em outros estudos. ${ }^{(13,15,22)}$

A cola cirúrgica não necessita de anestesia para a sua aplicação, sendo um procedimento rápido, indolor e de fácil execução por profissional capacitado. ${ }^{(13)}$ O Colégio Americano de Obstetras e Ginecologistas (The American College of Obstetricians and Gynecologists - ACOG) recomenda o uso da cola cirúrgica como uma opção para o reparo perineal de laceração de primeiro grau e da pele em laceraçóes de segundo grau, por apresentar menor tempo para o reparo, menor escore de dor referida e maior satisfação com o resultado (Recomendação nível B). ${ }^{(20)}$

Por se tratar de um estudo série de casos, houve limitação quanto ao pequeno tamanho da amostra, quanto à alta frequência de mulheres com laceraçóes de primeiro grau em comparação às com laceração de segundo grau e episiotomia. Esse fato decorreu da inclusão de mulheres no estudo por conveniência e não por critérios de randomização, como em um ensaio clínico. Ressalta-se que somente se procedeu ao reparo após a avaliação de um profissional do serviço, alheio à equipe de coleta, indicando a necessidade do procedimento.

Outra limitação observada foi a realização, por diferentes pesquisadoras, da avaliação dos escores das medidas, ao longo dos três momentos. Se, por um lado, esse fato viabilizou a execução da coleta no tempo adequado, diminuindo perdas de seguimento, por outro, talvez tenha possibilitado maior variação nos escores das medidas, decorrente de discordância entre as observadoras.

Em relação às dificuldades encontradas no desenvolvimento da técnica do uso da cola cirúrgica, verificou-se que a quantidade de cola utilizada para o reparo tecidual influenciava na realização adequada do procedimento, pois seu excesso impedia a aproximaçáo dos tecidos ao formar uma película não aderente. Essa dificuldade foi minimizada pela técnica gota a gota.

Outra dificuldade foi o sangramento excessivo nos tecidos a serem reparados, sendo contraindicado o uso da cola quando há necessidade de hemostasia local por pressão. A presença de sangramento excessivo também foi considerado como fator impeditivo para o uso da cola cirúrgica na literatura. ${ }^{(13)}$

$O$ presente estudo apresenta resultados que servirão de base para outras pesquisas. A aplicação da cola cirúrgica constitui uma opção à sutura tradicional e os resultados sugerem boa aceitação por parte das mulheres e bons resultados perineais. Considera-se importante que novos estudos sejam feitos sobre diferentes tipos de reparo perineal, objetivando encontrar os materiais e as técnicas que tragam os melhores benefícios para a mulher e para os profissionais que a assistem no parto.

\section{Conclusão}

A aplicação da cola cirúrgica mostrou-se viável para utilização em uma amostra maior de mulheres, mediante a técnica descrita neste estudo. Os resultados mostraram que nas primeiras 48 horas após o parto as mulheres tiveram dor de baixa intensidade ou ausente e que o processo de cicatrização foi favorável. As puérperas referiram satisfação com o reparo perineal, o que sugere boa aceitação da cola cirúrgica.

\section{Colaborações}

Caroci-Becker A, Brunelli WS, Lima MOP, Mendes EPB, Ochiai AM e Riesco MLG contribuíram com a concepção do estudo, análise e interpretação dos dados, redação do artigo, revisão crítica relevante do conteúdo intelectual e aprovação final da versão a ser publicada. 


\section{Referências}

1. Frohlich J, Kettle C. Perineal care. BMJ Clin Evid. 2015;2015:1401.

2. Oliveira LS, Brito LG, Quintana SM, Duarte G, Marcolin AC. Perineal trauma after vaginal delivery in healthy pregnant women. Sao Paulo Med J. 2014;132(4):231-8.

3. Naidu M, Sultan AH, Thakar R. Reducing obstetric anal sphincter injuries using perineal support: our preliminary experience. Int Urogynecol J Pelvic Floor Dysfunct. 2017;28(3):381-9.

4. Alvarenga MB, Francisco AA, Oliveira SM, Silva FM, Shimoda GT, Damiani LP. Avaliação da cicatrização da episiotomia: confiabilidade da escala REEDA (Redness, Oedema, Ecchymosis, Discharge, Approximation). Rev Lat Am Enfermagem. 2015;23(1):162-8.

5. Leeman L, Rogers R, Borders N, Teaf D, Qualls $C$. The effect of perineal lacerations on pelvic floor function and anatomy at 6 months postpartum in a prospective cohort of nulliparous women. Birth. 2016;43(4):293-302.

6. Kettle C, Dowswell T, Ismail KM. Continuous and interrupted suturing techniques for repair of episiotomy or second-degree tears. Cochrane Database Syst Rev. 2012 Nov 14;11(11):CD000947.

7. Aasheim V, Nilsen ABV, Reinar LM, Lukasse M. Perineal techniques during the second stage of labour for reducing perineal trauma. Cochrane Database Syst Rev. 2017 Jun 13;6(6):CD006672.

8. Leon-Larios F, Corrales-Gutierrez I, Casado-Mejía R, Suarez-Serrano C. Influence of a pelvic floor training programme to prevent perineal trauma: A quasi-randomised controlled trial. Midwifery. 2017;50:72-7.

9. Nguyen DH. Evidence Summary. Intrapartum: perineal and genital trauma (tears or episiotomy). Australia: The Joanna Briggs Institute EBP Database; 2017.

10. Dehne $T$, Zehbe $R$, Krüger JP, Petrova $A$, Valbuena $R$, Sittinger $M$, et al. A method to screen an devaluate tissue adhesives for joint repair applications. BMC Musculoskelet Disord. 2012;13:175.

11. García Cerdá D, Ballester AM, Aliena-Valero A, Carabén-Redaño A, Lloris JM. Use of cyanoacrylate adhesives in general surgery. Surg Today. 2015;45(8):939-56.
12. Neto JN, Assis AF. Aplicabilidade dos adesivos de cianoacrilato em feridas pós traumáticas e patológicas. Parte I. Rev Bras Cir Cabeça Pescoço. 2014;43(4):193-9.

13. Feigenberg T, Maor-Sagie E, Zivi E, Abu-Dia M, Ben-Meir A, Sela $H Y$, et al. Using adhesive glue to repair first degree perineal tears: a prospective randomized controlled trial. BioMed Res Int. 2014;2014:526590.

14. Teixeira TT, Caroci AS, Brunelli WS, Riesco ML. Tissue adhesive to repair first-degree perineal tears: a pilot randomized controlled trial. Clin Exp Obstet Gynecol. 2020;47(2):228-33.

15. Chamariya S, Prasad M, Chauhan A. Comparison of dermabond adhesive glue with skin suture for repair of episiotomy. Int J Reprod Contracept Obstet Gynecol. 2016;5:3461-5.

16. McCaffery M, Beebe A. Pain: clinical manual for nursing practice. St. Louis: Mosby; 1989. 353p.

17. Pimenta CA, Cruz DA, Santos JL. Instrumentos para avaliação da dor: 0 que há de novo em nosso meio? Braz Neurosurg. 1998;7(1):1524.

18. Davidson N. REEDA: evaluating postpartum healing. J Nurse Midwifery. 1974;19(2):6-8.

19. Metcalfe A, Tohill S. Perineal tear assessment and the development of the Peri-rule. In: Henderson C, Bick D, editors. Perineal care: an international issue. UK: Cromwell Press; 2005. p. 87-97.

20. Committee on Practice Bulletins-Obstetrics. ACOG Practice Bulletin No. 198: Prevention and Management of Obstetric Lacerations at Vaginal Delivery. Obstet Gynecol. 2018;132(3):e87-e102.

21. Jaiswal D, Wilkinson TR, Akhtar M. Cynoacrylate surgical glue as an alternative to suturing for mesh fixation in lichtenstein hernia repair. Int Surg J. 2018;5(5):1882-4.

22. Seijmonsbergen-Schermers AE, Sahami S, Lucas C, Jonge A. Nonsuturing or skin adhesives versus suturing of the perineal skin after childbirth: a systematic review. Birth. 2015;42(2):100-15.

23. Al-Abri R, Al-Balushi A. Patient satisfaction survey as a tool towards quality improvement. Oman Med J. 2014;29(1):3-7. 